\title{
Resources Students Use to Understand Quantum Mechanical Operators
}

\author{
Elizabeth Gire and Corinne Manogue
}

Department of Physics, Oregon State University, Corvallis, OR

\begin{abstract}
The Paradigms team at Oregon State University has developed a quantum mechanics curriculum aimed at middle division students that begins with a strong emphasis on using operators, matrices and Dirac notation to describe quantum systems. The curriculum begins with spin systems, and this content ordering relies on students being able to understand quantum mechanical operators, eigenstates and quantum measurement without prior instruction on wave functions. We have analyzed classroom and an interview video to identify resources students use when considering these quantum ideas. Identification of such resources will inform introductory curricula that are prerequisite to the quantum Paradigms and inform the development of Paradigms materials that will guide students to use these resources productively.
\end{abstract}

Keywords: cognitive resources, quantum mechanics, quantum measurement, eigenstates, operators

PACS: 01.40.Fk, 03.65.Xp

\section{INTRODUCTION}

The model of cognitive resources has been a powerful construct within the physics education community for understanding how students think and learn ${ }^{1,2}$. This model helps to explain how students are able to address unfamiliar problems and suggests an instructional method for guiding students to refine their intuition for productive formal analysis. For example, Elby ${ }^{3}$ discusses how students intuitively use a resource of "more agent results in more effect" in understanding collisions, and how reassigning this resource to consider accelerations rather than forces is a productive way of helping students to analyze collision phenomena. This instructional strategy emphasizes refining and redirecting intuition rather than abandoning incorrect preconceptions.

In classical physics, preconceptions are often assumed to be acquired through students' everyday experiences. In contrast, there is little opportunity for students to develop intuition specific to quantum mechanical phenomena in this way. Despite this fact, physics education researchers have documented misconceptions about quantum mechanics that seem to be common among students ${ }^{4,5}$. It is unlikely that these misconceptions form from daily experience. On the other hand, the resources model of cognition suggests that students may be using a common set of resources - resources that develop through everyday experiences and prior instruction in classical physics - when considering quantum mechanical phenomena. Reported misconceptions would then result from students' unproductive use of these resources.

In this study, we looked for resources students use for understanding the phenomenon of quantum measurement. We analyzed classroom video and video of a volunteer student working through a problem about quantum measurement. We find that some of the misconceptions that have been reported may be due to an inappropriate use of the resource "quantum measurement as agent." It is hoped that in identifying such resources, materials can be developed to either redirect how students use these resources or suggest the use of different resources for a more productive understanding of quantum measurement.

\section{RESEARCH ON QUANTUM MECHANICAL MISCONCEPTIONS}

Over the last fifteen years, many physics education researchers have turned their attention to advanced physics topics and courses. In the case of quantum mechanics, many misconceptions have been identified and have been shown to be shared by students with a variety of backgrounds. Some of the misconceptions having to do with quantum measurement include ${ }^{4,5}$ : 
- A measurement disturbs a quantum system (i.e. a measurement of a particle's position alters the value of its momentum)

- Given a system with a definite value of $L_{z}$, there exist definite values of $\mathrm{L}_{\mathrm{x}}$ and $\mathrm{L}_{\mathrm{y}}$ but they rotate so that when they are measured, the outcome of the measurement cannot be predicted.

- The equation $\mathrm{H} \Psi=\mathrm{E} \Psi$ is valid for all states $\Psi$ (not limited to eigenstates).

- $\mathrm{H} \Psi$ measures the energy of $\Psi$

In addition to the misconceptions that have been reported, Singh notes that "most advanced students [correctly] have some idea that the measurement of an observable collapses the wave function into an eigenstate of the corresponding operator, that a measurement on a system in a definite state could yield a multitude of results and that prior measurements affect future measurements." ${ }^{\circ} \mathrm{We}$ observe that students make statements consistent with these reports (although we do not take the view that the students' statements reflect them possessing unitary misconceptions but rather are the result of an unproductively applied resource).

\section{STUDY DESIGN AND CONTEXT}

The video that was used for this study was taken in the context of the three 2008 winter quarter Paradigms in Physics courses at Oregon State University ${ }^{7,8}$. Eighteen students were enrolled in each course, and nearly all students enrolled in all three courses. The courses are titled Spin, 1-D Waves, and Central Forces and are meant to be taken as a sequence. All of these courses are taken by junior-level students and include a significant amount of instruction in quantum mechanical phenomena, though Waves and Central Forces also include instruction in relevant classical physics. Prerequisites for these Paradigms courses include a sophomore level course in modern physics, a week long preface course in matrix methods, and a linear algebra course. ${ }^{9} \quad$ Author Gire was a coinstructor for all three of these Paradigms courses; Gire and Manogue taught Central Forces together.

We looked for group activities and whole class discussions that asked students to consider measurement questions that involve the use of quantum mechanical operators. Specifically, we noticed that many students mention that an operator acting on a state is a mathematical representation of making a quantum measurement. The examples presented in this paper illustrate the students' use of this idea. Students typically worked in groups of 3 or 4 students on tabletop whiteboards $(2 \mathrm{ft} \times 3 \mathrm{ft}$ ). Each group was filmed using a camera mounted on the wall looking down at the whiteboard with a microphone mounted on the table, in addition to a roaming camera that was able to capture whole class discussions.

A graduate student that had previously taken the Paradigms courses (pseudonym Oliver) volunteered to discuss his understanding of quantum measurement questions of this type. The specific question he considered was taken from an activity in the Central Forces Paradigm. He was filmed while he worked on a wall-mounted white board. Both authors were present at this unscripted, exploratory interview. This interview informed our understanding of student reasoning observed on the classroom videos.

\section{STUDENT REASONING EXAMPLES}

\section{Spins}

The following conversation began with a student's question about the eigenvalue equation associated with a spin operator. The student did not seem to recognize that only a special set of states satisfy the eigenvalue equation. The class was asked to consider the following equation $S_{x}|+\rangle=\hbar / 2|-\rangle$, and had determined that it is algebraically correct. The instructor then asked the unusual question "When in quantum mechanics might you write this equation down?" (The instructor later revealed that she intended for the students to try to assign some physical meaning to the equation, but did not expect the students to be successful).

Trevor: I bet it has something to do with the SternGerlach stuff. Um, well when, let's see...So we'll have particles getting through, spin up particles going through.... magnetic field aligned along the $\mathrm{z}$ axis first, making our prepared state, right, and then you do an operation where we're now pushing he particles through one along the $\mathrm{x}$ axis.

Instructor: Ok, I don't know: that's what you're proposing that this means. What do you think Robert?

Robert: Well, actually, that's what I was thinking, that's plus, that'd be your "in" and the $\mathrm{S}_{\mathrm{x}}$ will be operating on that plus ket. But of course, you already know (mumbles)

Instructor: What do you mean, it's not telling us what's coming out?

Robert: You're saying that you're putting in, well, the plus $\mathrm{x}$ in, the $\mathrm{S}_{\mathrm{x}}$ is (mumbles), that's why I'm getting that last part, and...

Instructor: Ok, so, does this equation tell me that what comes out, the state that comes out here is spin down? 
Robert: No (shakes his head).

Instructor: Samuel, what do you think?

Samuel: I just re-involved myself in the conversation, so...

Instructor: Ok, so the question is: we've figured, we wrote down this equation using some matrix algebra that corresponds to these operators and these states. So I have my spin in the $\mathrm{x}$ direction operator acting on my spin up in $\mathrm{z}$ direction ket, and it was proposed that this equation corresponds to this situation, where you send spin up in the $\mathrm{z}$ direction to a Stern-Gerlach that measures spin in the $\mathrm{x}$ direction, and the thing that you get out is spin down in the z-direction. What do you think about that?

Samuel: It um, that's how I would have initially interpreted it.

Instructor: Ok, initially, but...?

Samuel: But after some thought, I don't know.

Instructor: Ok, so you're not sure?

Samuel: No

Instructor: Ok. Joe, what do you think?

Joe: Well, I'm thinkin' that I don't see specifically any reason to say that that says there's an output necessarily. So, there's just something acting on an input.

Samuel: Spin operators do just correspond to measurements made like, they correspond to the act of making a measurement along that axis, right?

\section{Central Forces}

During the Central Forces Paradigm, students were asked to do an activity called "Quantum Calculations on a Ring". Each group is assigned a specific state of a particle confined to a ring and is asked to consider a series of questions, including: "If you measured the $\mathrm{z}$ component of angular momentum, what possible values could you obtain?" (Each state assigned was a superposition of angular momentum eigenstates. This particular group was considering the superposition state $\left.\Phi_{B}=\frac{1}{\sqrt{4 \pi r_{0}}}\left(e^{i \phi}+e^{2 i \phi}\right)\right)$

Matthew: So we have state B. By measuring...throw a Hamiltonian on it?

Samuel: If you measure the, don't we just have to operate the angular momentum operator on our state to find...? Is that the right way to think about it?

Matthew: I think so. It makes sense by the units is what it looks like. So, the angular momentum operator, negative i hbar, d by dt. Acting our state B. Ah...what possible values could you obtain?
Samuel: But how does that give us values?

Vincent: I don't know, but we'll act on it.

Joe: Now I don't know what that means, but...

Samuel: Does it mean, so, what possible values could you obtain, so this gives us possible values based on phi. Does that...? So our different values are just...

Matthew: The $\mathrm{z}$ component is how the state changes...

Samuel: No, that's not what I mean. (now addressing the instructor) It's asking like, what possible values could you obtain? Does that mean given, to get the values, different values it's just for different values of phi?

Instructor: No. Is that what it looks like?

Matthew: The state of phi depends on phi.

Joe: Can we try it like that?

Instructor: So in general, when you measure a state, what values can you get out?

Joe: Up or down.

Instructor: For spin, yes.

Samuel: The eigenvalues of the operator?

Instructor: The eigenvalues of the operator are the possible values that you could measure.

Samuel: So this, this is not making any sense?

Instructor: Why doesn't it make sense?

Samuel: I don't know, I thought it did make sense.

Instructor: Well, why doesn't it make sense to you?

Samuel: Well it seems like continuous. Can you get a continuous...

Matthew: It's not a discreet value.

Samuel: Yeah. Oh, so its eigenvalues are superpositions of eigenvalues.

Instructor: It's not a superposition of eigenvalues.

Samuel: So, when I wrote L, I just meant angular momentum.

Instructor: You meant this, right?

Samuel: No, I meant, I was saying was that the angular momentum is equal to the angular momentum operator acting on a state.

Instructor: Is that true?

Samuel: Not necessarily.

Matthew: Sure, but it looked like something we could do.

Samuel: That's why I was asking if it made sense rather than stating whether or not it did.

\section{Oliver}

Oliver was asked to consider the same question from the "Quantum Calculations on a Ring" activity. Oliver had let the angular momentum operator act on each term of his initial state function, but felt the resulting coefficients did not make sense. 
Oliver: 'Cause it seems like I should be able to just measure $\mathrm{L}_{\mathrm{z}}$. Right, I've got this $\mathrm{L}_{\mathrm{z}}$ operator, $\boldsymbol{I}$ should just be able to operate on the function and $L_{z}$ is like my measuring device. Because if I have $S_{z}$ or whatever that's my Stern-Gerlach device. And so if I've got $L_{z}$ that's my measuring device. But if I've got the $S_{z}$ one, let's back to that. So if I have, if I'm measuring $S_{z}$ on something. So then I have, I'm measuring $S_{z}$ on some plus state or something...

Oliver goes on to discuss measurements of spin. When he gets stuck, the interviewer then redirects his attention to his previous statements.

Interviewer: I want to take you back to a statement you made a few minutes ago, which was you said $\ldots \mathrm{S}_{\mathrm{z}}$ was your measuring device.

Oliver: Yes.

Interviewer: Why do you say that?

Oliver: Um, it operates, it's transforming the state from one thing to another, and we're representing the, what happens inside that Stern-Gerlach device to the state vector with this matrix, so this is telling me, this is representing the mathematical operations on the state that occurred inside that device.

Interviewer: Why do you believe that?

Oliver: I've thought it through. I would need to rethink it through... So I'm sending my beam in, and it goes into my Stern-Gerlach and it's going to split out in two directions... And, the little dots I get on the screen here from the particles being there, are indicative of particles that had either $\mathrm{hbar} / 2$ spin or $+\mathrm{hbar} / 2$ spin along the axes I'm measuring. And so this takes whatever vector or whatever state I have and forces it to say I'm only looking at what's happening along that axis.

\section{DISCUSSION}

Each of the examples above includes students stating a belief (sometimes a tentative belief) that when an operator acts on a state it is a mathematical representation of making a measurement of the physical quantity associated with the operator. We have observed that comments to this effect are common in our quantum mechanics courses. These comments, and several of the misconceptions about quantum measurements that have been reported, may be due to students activating a resource of "quantum measurement as an agent" - that is, making a quantum measurements changes the system. This resource could be applied productively in the context of a

measurement causing the wavefunction to collapse into an eigenstate (a physical phenomenon that does not follow directly from the mathematical tools of quantum mechanics, but rather must be put into quantum calculations by hand). This resource seems to be similar to the resource of "operator as agent"; students seem to be aware that matrices (operators) acting on vectors generally causes a transformation of the vector. Since students also know that operators have a correspondence with measurable quantities, the activation of this resource, although unproductive in the context of these problems, is quite understandable.

One instructional strategy may be to guide students away from this inappropriate resource and encourage the activation of a "quantum measurement as selector" resource - that is, performing a quantum measurement results in the selection of an eigenstate that was a piece of the linear expansion of the original state.

\section{ACKNOWLEDGMENTS}

We'd like to extend our gratitude to Leonard Cerny, Gulden Karakok, David McIntyre, Janet Tate and Dedra Demaree for many helpful conversations about student reasoning, and to the Paradigms students who have generously tolerated being filmed throughout their Paradigms experience. This material is based upon work supported by the National Science Foundation under DUE Grant No. 0618877.

\section{REFERENCES}

D. Hammer, A. Elby, R. Scherr, and F. R. Edward, in Transfer of Learning from a Modern Multidisciplinary Perspective, edited by J. Mestre (Information Age Publisher, Greenwich, CT, 2005), p. 89.

2 D. Hammer and A. Elby, Journal of the Learning Sciences 12, 53 (2003).

3 A. Elby, American Journal of Physics 69, S54 (2001).

4 D. F. Styer, American Journal of Physics 64, 31 (1996).

5 C. Singh, M. Belloni, and W. Christian, Physics Today 59, 43 (2006).

6 C. Singh, American Journal of Physics 69, 885 (2001).

7 C. A. Manogue, P. J. Siemens, J. Tate, K. Browne, M. L. Niess, and A. J. Wolfer, American Journal of Physics 69, 978 (2001).

8 Paradigms material can be found at the Portfolios Wiki http://www.physics.oregonstate.edu/portfolioswiki/

9 OSU Course Catalog, accessed June 2008 http://catalog.oregonstate.edu/CourseList.aspx?subject $\underline{\text { code }=\text { PH\&level }=\text { undergrad } \& \text { campus }=\text { corvallis }}$ 